\title{
Hyperspectral based discrimination of thyroid and parathyroid during surgery
}

Manuel Barberio, Institute of Image-Guided Surgery (IHU), University of Strasbourg, Strasbourg, France, manuel.barberio@ihu-strasbourg.eu

Marianne Maktabi, Innovation Center Computer Assisted Surgery (ICCAS), University of Leipzig, Leipzig, Germany, marianne.maktabi@medizin.uni-leipzig.de

Ines Gockel, Department of visceral, thorax, transplantation and vascular surgery, University Hospital of Leipzig, Leipzig, Germany, ines.gockel@medizin.uni-leipzig.de

Nada Rayes, Department of visceral, thorax, transplantation and vascular surgery, University Hospital of Leipzig, Leipzig, Germany, nada.rayes@medizin.uni-leipzig.de

Boris Jansen-Winkeln, Department of visceral, thorax, transplantation and vascular surgery, University Hospital of Leipzig, Leipzig, Germany, boris.jansen-winkeln@medizin.uni-leipzig.de

Hannes Köhler, Innovation Center Computer Assisted Surgery (ICCAS), University of Leipzig, Leipzig, Germany, hannes.koehler2@medizin.uni-leipzig.de

Sebastian M Rabe, Department of visceral, thorax, transplantation and vascular surgery, University Hospital of Leipzig, Leipzig, Germany, sebastianmurad.rabe@medizin.uni-leipzig.de

Lena Seidemann, Department of visceral, thorax, transplantation and vascular surgery, University Hospital of Leipzig, Leipzig, Germany, lena.seidemann@medizin.uni-leipzig.de

Jonathan P Takoh, Department of visceral, thorax, transplantation and vascular surgery, University Hospital of Leipzig, Leipzig, Germany, jonathanphilip.takoh@medizin.uni-leipzig.de

Michele Diana, Institute of Image-Guided Surgery (IHU), University of Strasbourg, Strasbourg, France, michele.diana@ihu-strasbourg.eu

Thomas Neumuth, Innovation Center Computer Assisted Surgery (ICCAS), University of Leipzig, Leipzig, Germany, thomas.neumuth@medizin.uni-leipzig.de

Claire Chalopin, Innovation Center Computer Assisted Surgery (ICCAS), University of Leipzig, Leipzig, Germany, claire.chalopin@medizin.uni-leipzig.de

The number of incidental parathyroidectomies during endocrine neck procedures remains even in specialized endocrine centers around $16 \%$. Therefore, there is a critical need for an intraoperative method for parathyroid identification in real time. Hyperspectral imaging (HSI) is a new approach in the medical area which provides simultaneously spatial and spectroscopic information. It represents a promising approach to intraoperatively discriminate the parathyroids from other structures.

The HSI system TIVITA (500-1000 nm) was used to acquire absorbance spectra of the parathyroids and thyroids during endocrine neck surgeries of seven patients. Both glands were annotated in the HIS data and the mean normalized absorption spectra in the ROIs generated. Then the averaged spectra and the standard deviations were computed on the set of patients. They were visually analyzed to extract specific spectral signatures for both glands.

A total of 19 datasets for seven patients were acquired. Four of them were removed because of impair of the sperctra. The mean absorbance spectra of both glands showed differences in the range between 600 and $700 \mathrm{~nm}$ and at 760 and $960 \mathrm{~nm}$. This means that thyroid and parathyroid have different oxygenation states and different contents of deoxygenated hemoglobin and water. From these observations, it is possible to define spectral signatures to characterize both glands. We showed on one patient data set how spectral signatures can be integrated in classification algorithms to automatically identify the thyroid and parathyroid from other structures. The classification approach uses decision forest based on the second derivatives of the absorbance spectra. A visual discrimination of the parathyroid was obtained.

The extraction of spectral features of the parathyroid and thyroid using an HSI system has proven to be feasible. Those features can be used to discriminate the glands from the surrounding structures. 


\section{Minimally invasive mastoidectomy approach using a mouldable surgical target- ing system. A proof of concept.}

Thomas S. Rau, Department of Otolaryngology and Cluster of Excellence EXC 1077/1 “Hearing4all”, Hannover Medical School, Carl-Neuberg-Str. 1, 30625 Hannover, Germany, rau.thomas@mh-hannover.de

Sina Witte, Department of Otolaryngology and Cluster of Excellence EXC 1077/1 “Hearing4all”, Hannover Medical School, Carl-Neuberg-Str. 1, 30625 Hannover, Germany, witte.sina@stud.mh-hannover.de

Lea Uhlenbusch, Department of Otolaryngology and Cluster of Excellence EXC 1077/1 “Hearing4all”, Hannover Medical School, Carl-Neuberg-Str. 1, 30625 Hannover, Germany, Leauhlenbusch@gmx.de

G. Jakob Lexow, Department of Otolaryngology and Cluster of Excellence EXC 1077/1 “Hearing4all”, Hannover Medical School, Carl-Neuberg-Str. 1, 30625 Hannover, Germany, Lexow.jakob@mh-hannover.de

Silke Hügl, Department of Otolaryngology and Cluster of Excellence EXC 1077/1 “Hearing4all”, Hannover Medical School, Carl-Neuberg-Str. 1, 30625 Hannover, Germany, huegl.silke@mh-hannover.de

Lüder A. Kahrs, Institute of Mechatronic Systems, Leibniz Universität Hannover, Applestr. 11a, 30167 Hannover, Germany, Lueder.Kahrs@imes.uni-hannover.de

Omid Majdani, Department of Otolaryngology and Cluster of Excellence EXC 1077/1 “Hearing4all”, Hannover Medical School, Carl-Neuberg-Str. 1, 30625 Hannover, Germany, Majdani.omid@mh-hannover.de

Thomas Lenarz, Department of Otolaryngology and Cluster of Excellence EXC 1077/1 “Hearing4all”, Hannover Medical School, Carl-Neuberg-Str. 1, 30625 Hannover, Germany, Lenarz.thomas@mh-hannover.de

Hearing restoration using a cochlear implant requires a surgical access to the inner ear. In order to enhance patient safety, reduce trauma, and shorten the patient's time under anaesthesia current research focusses on minimally invasive cochlear implantation surgery by drilling only a single bore hole. This demands a highly accurate surgical assistance device to guide the drill along a predetermined trajectory planned in patient's image data. In this study a recently developed surgical targeting system was evaluated for the first time in a human cadaver trial. After screwing a reference frame on a temporal bone specimen and imaging of both, a trajectory through the facial recess was planned in order to reach the middle ear. Based on this plan a patient specific surgical template including a linear guide for the surgical drill was composed utilizing bone cement. After the hardening of the bone cement the surgical template was mounted on top of the reference frame. The drilling could be performed as previously planned without harming facial nerve and chorda tympani. The deviation of the actual drill hole to the planned trajectory was $0.17 \mathrm{~mm}$ at the level of the facial recess. The minimal distance of the drill hole to the facial nerve was $0.59 \mathrm{~mm}$. This proof-of-concept study demonstrates the feasibility of performing the access to the middle ear in a minimally invasive manner using the mouldable surgical targeting system. The presented process allows the patient specific individualization of a drill guide under sterile conditions. This might facilitate its integration into clinical routine. 


\title{
Surgical Tool Classification in Laparoscopic Videos Using Convolutional Neural Network
}

Tamer Abdulbaki Alshirbaji, Medical and life science, Furtwangen University, Institute of Technical Medicine, Villingen-Schwenningen, Germany, e-mail: abd@hs-furtwangen.de

Nour Aldeen Jalal, Medical and life science, Furtwangen University, Institute of Technical Medicine, VillingenSchwenningen, Germany, e-mail: ja@hs-furtwangen.de

Knut Möller, Medical and life science, Furtwangen University, Institute of Technical Medicine, VillingenSchwenningen, Germany, e-mail: moe@hs-furtwangen.de

\begin{abstract}
Laparoscopic videos are a very important source of information which is inherently available in minimally invasive surgeries. Detecting surgical tools based on that videos have gained increasing interest due to its importance in developing a context-aware system. Such system can provide guidance assistance to the surgical team and optimise the processes inside the operating room. Convolutional neural network is a robust method to learn discriminative visual features and classify objects. As it expects a uniform distribution of data over classes, it fails to identify classes which are under-presented in the training data. In this work, loss-sensitive learning approach and resampling techniques were applied to counter the negative effects of imbalanced laparoscopic data on training the CNN model. The obtained results showed improvement in the classification performance especially for detecting surgical tools which are shortly used in the procedure.
\end{abstract}




\section{Suggested mandibular reconstruction for computer-assisted planning of bone grafts with autologous fibula segments}

Niclas Hagen, Institute of Medical Biometry and Informatics, University of Heidelberg, Heidelberg, Germany, niclas.hagen@med.uni-heidelberg.de

Urs Eisenmann, Institute of Medical Biometry and Informatics, University of Heidelberg, Heidelberg, Germany

Sebastian Kallus, Institute of Medical Biometry and Informatics, University of Heidelberg, Heidelberg, Germany

Christian Freudlsperger, Department of Oral and Maxillofacial Surgery, Heidelberg University Hospital, Heidelberg, Germany

Hartmut Dickhaus, Institute of Medical Biometry and Informatics, University of Heidelberg, Heidelberg, Germany

Tumor-related mandibular reconstructions by autologous fibula flaps are of high importance in maxillofacial surgery. Computer-assisted planning for this purpose is generally accepted to obtain a better functional and aesthetic outcome but can be very time consuming because of several interactive tasks involved. Therefore, our aim is to automatically calculate a suggested reconstruction for the bone graft and the underlying configuration of the fibula segments to reduce the accruing planning effort.

In cooperation with Heidelberg University Hospital, we develop a computer-based planning workflow for mandibular reconstructions, including segmentation, resection planning and 3D-reconstruction of the graft. The planning tool is based on the open source platform "Medical Imaging Interaction Toolkit" (MITK). After resection planning, the tool automatically calculates a suggested reconstruction considering patient-specific geometrical information and surgical knowledge. Most important target figures are:

- Optimizing cutting angels between adjacent fibula segments to obtain high contact surfaces

- Restoring the shape of the natural mandible

- Ensuring the correct course of the providing blood vessel

Finally, the surgeon may interactively fine-tune the planning result if necessary.

The planning results can be utilized in different ways. The involved components (mandible, graft and underlying fibula segments) may be prepared for 3D-printing. The resulting 3D-models are used by orthodontists to prepare patient-specific cutting guides and predefined reconstruction plates. Furthermore, we defined an interface to use the planning results for intraoperative electromagnetic navigation, which is also developed by our group. We initially tested our approach by performing the whole planning procedure regarding four patient cases. The overall planning procedure took less than 5 minutes. The automatic suggested reconstructions were rated to be a good approximation by the surgeons. Final planning results were archived after fine-tuning the graft segments $(2.2 \mathrm{~mm} \pm 2.1 \mathrm{~mm})$ in about 2.5 minutes. The final results were rated as optimal in each case. 


\section{Modular design of versatile surgical mini-robots - Approach towards a better cost-to-benefit ratio in surgical robotics}

Lukas Theisgen, Chair of Medical Engineering at Helmholtz-Institute for Biomedical Engineering of RWTH Aachen University, Aachen, Germany, theisgen@hia.rwth-aachen.de

Matías de la Fuente, Chair of Medical Engineering at Helmholtz-Institute for Biomedical Engineering of RWTH Aachen University, Aachen, Germany, fuente@hia.rwth-aachen.de

Klaus Radermacher, Chair of Medical Engineering at Helmholtz-Institute for Biomedical Engineering of RWTH Aachen University, Aachen, Germany, radermacher@hia.rwth-aachen.de

Surgical robots have been introduced in the field of Computer Assisted Surgery (CAS) to assist the surgeon by providing an accurate link between the computer-based plan and the exact (a) positioning or (b) dynamic path control of an instrument on the operating site respectively. Whereas initial systems mostly have been based on an active supervisory control scheme of industrial robots with large universal workspaces, later on specialized miniaturized kinematics have been proposed, with restricted workspaces adapted to specific applications in order to ease handling and provide inherent safety properties. However, this specialization resulted in even narrower fields of application, low quantities and higher costs. Modularization seems to be a key factor to combine the benefits of both approaches. In this paper two modular robotic solutions, validated in the context of their purpose, are analysed regarding their modular design. Based on this, the potential of modularity for instrument guiding tasks in surgery is discussed. 


\section{Evaluating Convolutional Neural Network and Hidden Markov Model for Rec- ognising Surgical Phases in Sigmoid Resection}

Nour Aldeen Jalal, Furtwangen University, Institute of Technical Medicine, Villingen-Schwenningen, Germany, eMail: ja@hs-furtwangen.de

Tamer Abdulbaki Alshirbaji, Knut Möller, Furtwangen University, Institute of Technical Medicine, VillingenSchwenningen, Germany, e-Mail: \{abd,moe\}@hs-furtwangen.de

Surgical workflow analysis in laparoscopic surgeries has been studied widely during last years because of its various applications. For example, optimising the schedule of operating rooms (OR) and developing a context-aware system that supports surgical team during the intervention. Surgical phase recognition has been applied to various kinds of laparoscopic procedures, mainly of type cholecystectomy. Sigmoid resection procedures are considered more complex than cholecystectomy, and they have not been extensively studied. Therefore, the focus of this work is to study phase recognition in sigmoid resection. In this paper, a convolutional neural network (CNN) architecture and Hidden Markov Model (HMM) were evaluated for performing phase recognition in sigmoid resection videos. The CNN is an extension of a pre-trained model, and it was fine-tuned to perform the recognition. To consider the temporal aspect of the phase sequences, confidences obtained by the CNN were then provided into a HMM to release final classification. Experimental results show a low performance of the proposed method to recognise surgical phases in such complex procedures. Therefore, the dataset used for the evaluation was also reviewed, and statistics of each phase were generated. 


\section{Towards acoustic emission and ultrasound integration for needle guidance in minimally invasive procedures}

Laveena Kewlani, Computer Aided Medical Procedures \&Augmented Reality, Technische Universität München, München, Germany, laveena.kewlani@tum.de Alfredo Guillermo Illanes Manriquez, INKA-Intelligente Katheter, Otto von Guericke University, Madgeburg, Germany, alfredo.illanes@ovgu.de

Ivan Maldonado Zambrano, INKA-Intelligente Katheter, Otto von Guericke University, Madgeburg, Germany, ivan.maldonado@ovgu.de

Bjoern Menze, Chair of Image-Based Biomedical Modeling, Technische Universität München, München, Germany, bjoern.menze@tum.de

Michael Friebe, INKA-Intelligente Katheter, Otto von Guericke University, Madgeburg, Germany, michael.friebe@ovgu.de

Medical Interventional devices (MIDs) such as needles, catheter and guide wires are challenging to use during interventional surgeries due to interrupted visualization and artefacts. Therefore, MIDs need highly efficient accessories to allow targeting and verification simultaneously. Diagnostic imaging devices (CT, Ultrasound, X-rays and MRI) are often used to suffice this need, but only visual perception is sometimes not sufficient due to several imaging related shortcomings like noise, poor visibility within the slice or volume, material related artefacts, incorrect positioning etc. Although ultrasound is extensively used for tracking MIDs in minimal invasive surgeries (MIS) common acoustic and anatomical artefacts are hard to handle manually.

We propose a novel technological approach that reads acoustic signals via a sound receiver attached to the proximal end of a needle to support the MIDs verification and tracking during interventional surgeries. In addition, we developed and introduced a needle tracking algorithm for ultrasound B-mode images that also supports the intervention visually. The algorithm acquires the acoustic signal from the interaction of the needle with tissue during insertion and simultaneously detects the needle in the ultrasound images using optical flow and progressive regional properties. The sampling frequency of the audio signal is $44.100 \mathrm{~Hz}$ with an acquisition frame rate of approximately 21 frames per second for the ultrasound after processing the data in real time. The proposed combination has the potential to solve some of the problems associated with needle localization and can provide tissue classification information in clinical procedures like biopsy, regional anesthesia, central venous catherization etc., where its crucial to track series of event for verification and target location. 